\title{
Outcomes after Extracorporeal Membrane Oxygenation in Neonates with Congenital Diaphragmatic Hernia: A Single- Center Experience
}

\author{
Wooseok Choi, M.D. ${ }^{1,}$, Won Chul Cho, M.D., Ph.D. ${ }^{2,}$, Eun Seok Choi, M.D., Ph.D. ${ }^{1}$, Tae-Jin Yun, M.D., Ph.D. ${ }^{1}$ \\ Chun Soo Park, M.D., Ph.D. ${ }^{1}$
}

'Division of Pediatric Cardiac Surgery, Department of Thoracic and Cardiovascular Surgery, Asan Medical Center, University of Ulsan College of Medicine, Seoul; ${ }^{2}$ Department of Thoracic and Cardiovascular Surgery, Gangneung Asan Hospital, University of Ulsan College of Medicine, Gangneung, Korea

\author{
ARTICLE INFO \\ Received May 11, 2021 \\ Revised September 6, 2021 \\ Accepted September 8, 2021 \\ Corresponding author \\ Chun Soo Park \\ Tel 82-2-3010-3583 \\ Fax 82-2-3010-6966 \\ E-mail chunsoo@amc.seoul.kr \\ ORCID \\ https://orcid.org/0000-0001-8718-8904 \\ *These authors equally contributed to this \\ work.
}

\begin{abstract}
Background: Congenital diaphragmatic hernia $(\mathrm{CDH})$ is a rare disease often requiring mechanical ventilation after birth. In severe cases, extracorporeal membrane oxygenation (ECMO) may be needed. This study analyzed the outcomes of patients with CDH treated with ECMO and investigated factors related to in-hospital mortality.

Methods: Among 254 newborns diagnosed with CDH between 2008 and 2020, 51 patients needed ECMO support. At Asan Medical Center, a multidisciplinary team approach has been applied for managing newborns with CDH since 2018. Outcomes were compared between hospital survivors and nonsurvivors.

Results: ECMO was established at a median of 17 hours after birth. The mean birth weight was $3.1 \pm 0.5 \mathrm{~kg}$. Twenty-three patients (23/51, 45.1\%) were weaned from ECMO, and 16 patients $(16 / 51,31.4 \%)$ survived to discharge. The ECMO mode was veno-venous in 24 patients (47.1\%) and veno-arterial in 27 patients (52.9\%). Most cannulations (50/51, 98\%) were accomplished through a transverse cervical incision. No significant between-group differences in baseline characteristics and prenatal indices were observed. The oxygenation index (1 hour before: 90.0 vs. 51.0, p=0.005) and blood lactate level (peak: 7.9 vs. 5.2 $\mathrm{mmol} / \mathrm{L}, \mathrm{p}=0.023$ ) before ECMO were higher in nonsurvivors. Major bleeding during ECMO more frequently occurred in nonsurvivors (57.1\% vs. $12.5 \%, p=0.007$ ). In the multivariate analysis, the oxygenation index measured at 1 hour before ECMO initiation was identified as a significant risk factor for in-hospital mortality (odds ratio, 1.02; 95\% confidence interval, 1.01-1.04; $p=0.05$ ).

Conclusion: The survival of neonates after ECMO for $\mathrm{CDH}$ is suboptimal. Timely application of ECMO is crucial for better survival outcomes.
\end{abstract}

Keywords: Congenital diaphragmatic hernias, Extracorporeal membrane oxygenation, Oxygenation index, Pediatric, Extracorporeal circulation

\section{Introduction}

Congenital diaphragmatic hernia $(\mathrm{CDH})$ is characterized by a congenital defect in the diaphragm and herniation of the abdominal organs into the thoracic cavity. It occurs in 1 of 3,300 live births [1]. Lung hypoplasia and persistent pulmonary hypertension lead to respiratory failure, resulting in clinical deterioration. Recently, in addition to the application of pulmonary vasodilators and high-frequency oscillatory ventilation (HFOV), management strategies that minimize ventilator-associated lung injury have significantly improved outcomes [1]. However, extracorporeal membrane oxygenation (ECMO) is required in patients with low preductal oxygen saturation, severe hypoxemia, severe hypercapnia, and insufficient oxygen delivery despite maximal ventilatory and pharmacological support.

In the Extracorporeal Life Support Organization (ELSO) database, $\mathrm{CDH}$ is the most common indication for neonatal ECMO. Since 1987, when the ELSO database was established, 7,889 cases of ECMO for $\mathrm{CDH}$ have been registered, 
with a survival rate of $50 \%$. However, no improvement in the survival rate has been achieved, and the role of ECMO in patients with $\mathrm{CDH}$ remains controversial [2]. Nevertheless, the necessity of ECMO in patients with certain severely detrimental conditions is unquestionable. This study was conducted to investigate the outcomes of ECMO in neonates with $\mathrm{CDH}$ and to identify factors associated with in-hospital mortality.

\section{Methods}

\section{Patient selection and definitions}

From April 2008 through May 2020, among 254 neonates with $\mathrm{CDH}, 51$ patients who were treated with ECMO were included in this study (Fig. 1). Data were collected and collated by reviewing electronic medical records. The birth profiles, fetal ultrasound data, and clinical data were obtained. Laboratory data, including serum lactate levels and the oxygenation index (OI; OI=mean airway pressure $\times \mathrm{FiO}_{2}$ [fraction of inspired $\left.\mathrm{O}_{2}\right] \times 100 \div \mathrm{PaO}_{2}$ ) were serially obtained 6 and 12 hours after birth and 1 hour before ECMO initiation. Surgical data with respect to ECMO establishment and hernia repair were collected. Follow-up data including respiratory and neurodevelopmental status were also obtained.

The primary outcome of interest was in-hospital mortality. For comparison, the patients were divided into 2 groups based on hospital survival: the survivor group and the nonsurvivor group. Major surgical bleeding during ECMO was defined as bleeding that required surgical intervention, and major medical bleeding included intracranial hemorrhage or pulmonary hemorrhage that needed medical management. The institutional review board of Asan Medical Center approved this study and granted a waiver of informed consent (study no., 2020-1461).

\section{Medical management and indication of extracorporeal membrane oxygenation}

The patients with $\mathrm{CDH}$ were immediately resuscitated after birth by an attending neonatologist. HFOV was considered when conventional mechanical ventilation failed to stabilize the baby (peak airway pressure $>25 \mathrm{cmH}_{2} \mathrm{O}$ ). Inhaled nitric oxide (NO) was applied when respiratory failure occurred despite HFOV (mean airway pressure 16-22 $\mathrm{cmH}_{2} \mathrm{O}$ ), in the presence of evidence of pulmonary hypertension. Transthoracic echocardiography was performed to check for any combined cardiac anomalies and to assess
Congenital diaphragmatic hernia $(n=254)$

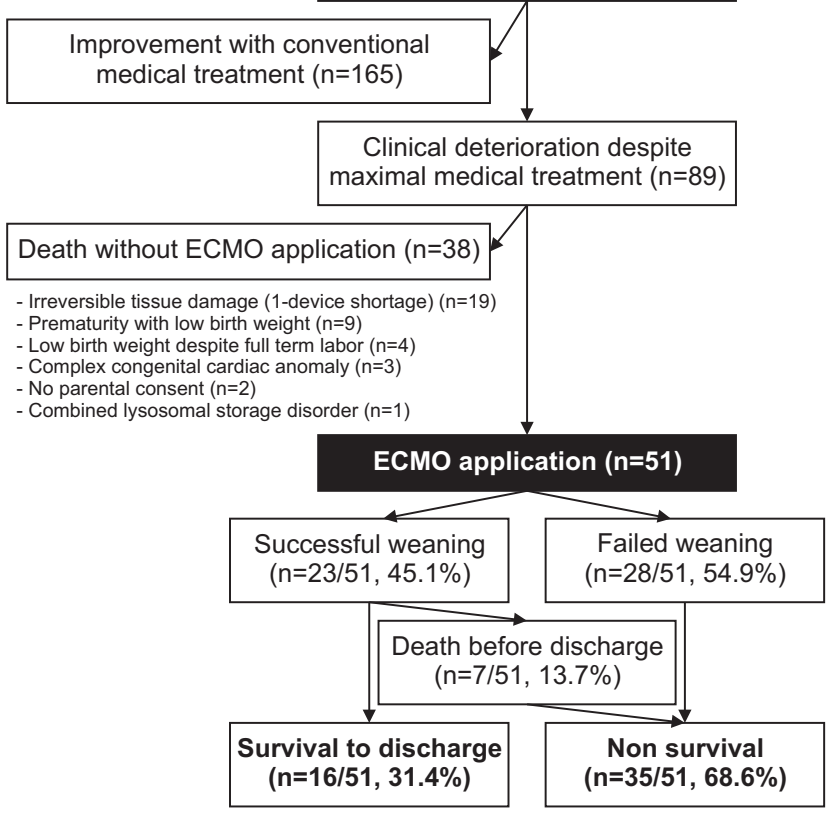

Fig. 1. Flow diagram showing the patient inclusion process. ECMO, extracorporeal membrane oxygenation.

the degree of pulmonary hypertension and subsequent ventricular dysfunction. Extracardiac congenital defects and chromosomal abnormalities were thoroughly assessed. ECMO was considered when the OI did not improve (OI > 40) despite maximum medical support, such as HFOV and inhaled NO. Some conditions precluded the application of ECMO, such as various chromosomal anomalies, other concomitant congenital defects that could affect survival, prematurity ( $<34$ weeks of gestation), low body weight $(<2$ $\mathrm{kg}$ ), and existing irreversible major organ damage (Fig. 1).

\section{Surgical technique and management of extracorporeal membrane oxygenation}

ECMO was established by a pediatric cardiac surgery team, usually within 1 hour after the initial notification. The mode of ECMO was decided by the surgeon. Prior to delivery, the surgical team was informed about newborns who had been prenatally diagnosed with $\mathrm{CDH}$. In most patients, the right common carotid artery and right internal jugular vein were exposed through a transverse cervical incision for arterial and venous cannulation.

The priming solution for the ECMO circuit comprises plasma solution, 20\% albumin, and packed red blood cells. For veno-arterial ECMO, a 10F arterial cannula (DLP Pe- 
diatric One-Piece Arterial Cannula; Medtronic, Fridley, MN, USA), and a $14 \mathrm{~F}$ venous cannula (DLP Single Stage Venous Cannula; Medtronic) was usually used. For veno-venous ECMO, a 13F dual-lumen cannula (OriGen Biomedical, Austin, TX, USA) was used. The flow rate was adjusted based on the degree of pulmonary or cardiac support.

Anticoagulation with unfractionated heparin was initiated once complete hemostasis was confirmed. The target range of activated clotting time and activated partial thromboplastin time on ECMO were 150-180 seconds and 50-70 seconds, respectively. The level of hematocrit should be maintained above 35\% and a platelet count should be kept at least $100,000 / \mu \mathrm{L}$. Ventilatory support was minimized to alleviate associated lung injury. Decannulation could be considered if the level of oxygen or carbon dioxide was adequate without any evidence of low cardiac output after 1-2 hours of interruption of gas flow in veno-venous ECMO or after longer than 30 minutes of cessation of ECMO flow in veno-arterial ECMO. To prevent circuit thrombosis, re-circulation of the ECMO circuit and intermittent flushing through arterial and venous cannulae were essential.

\section{Hernia repair}

Surgical repair of the diaphragmatic hernia was performed by a dedicated team from the Division of Pediatric Surgery. The defect was closed either primarily or using prosthetic patches including polytetrafluoroethylene with thickness of $0.5 \mathrm{~mm}$ (W. L. Gore \& Associates Inc., Flagstaff, AZ, USA), tissue collagen matrix with thickness of 1.5 $\mathrm{mm}$ (Tissue Science Laboratory, Covington, USA), and extracellular matrix biologic tissue matrix patches (Depuy Synthes, Oberdorf, Switzerland). Hernia repair was performed during or after ECMO at the surgeon's discretion.

\section{Multidisciplinary approach}

At our center, a multidisciplinary team approach has been applied for all neonates with CDH since 2018. Obstetricians shared information about the delivery schedule of babies with $\mathrm{CDH}$ to neonatologists, who subsequently informed the pediatric cardiac surgery team of the need to prepare for ECMO. The treatment of babies with $\mathrm{CDH}$ was conducted by neonatologists, and all options (e.g., medications, ventilatory support, and ECMO support) were offered in the neonatal intensive care unit. The timing of initiation or cessation of ECMO was determined based on the criteria developed by the multidisciplinary team. A standardized management protocol was implemented during ECMO.

\section{Statistical analysis}

Continuous variables are expressed as mean with standard deviation or median with interquartile range. Comparisons were performed using the Student t-test for data with a normal distribution and the Mann-Whitney U-test for skewed data based on the normality test. The normality of all continuous variables was checked visually and using the Shapiro-Wilk test.

The risk factors for in-hospital mortality were evaluated using logistic regression analysis. Variables found to have a p-value less than 0.1 in the univariable model were included in the multiple logistic regression models. A value of $\mathrm{p}<0.05$ was considered to indicate statistical significance. All analyses were performed using R software ver. 3.3.2 (R Foundation for Statistical Computing, Vienna, Austria).

\section{Results}

\section{Overall outcomes}

Among 51 patients with $\mathrm{CDH}$ requiring ECMO, 23 patients $(23 / 51,45.1 \%)$ were successfully weaned from ECMO, and 16 patients $(16 / 51,31.4 \%)$ survived to discharge (Fig. 1). Seven patients who could be weaned from ECMO died. The causes of death and intervals from decannulation to death in those patients are summarized in Table 1.

\section{Prenatal measures, baseline characteristics, and clinical variables prior to $\mathrm{ECMO}$}

Table 2 summarizes the baseline characteristics and prenatal sonographic measures of the patients treated with ECMO. There was no difference in any of the variables between survivors and nonsurvivors.

Table 3 summarizes the clinical variables before ECMO initiation. Echocardiographic data showed no left-to-right shunt through a patent ductus arteriosus on initial echocardiography before ECMO application. The proportion of right-to-left shunt and bidirectional shunt showed no statistically significant difference between the 2 groups. Before the initiation of ECMO, the initial lactate level (3.2 versus $5.8 \mathrm{mmol} / \mathrm{L}, \mathrm{p}=0.048)$ and peak lactate level $(5.2$ versus $7.9 \mathrm{mmol} / \mathrm{L}, \mathrm{p}=0.023$ ) were significantly lower in the survivor group than in the nonsurvivor group. The OI at 6 
Table 1. Causes of death for patients who died after extracorporeal membrane oxygenation weaning

\begin{tabular}{llccl}
\hline & Sex & $\begin{array}{c}\text { Weaning to } \\
\text { death (day) }\end{array}$ & $\begin{array}{c}\text { Age at } \\
\text { death (day) }\end{array}$ & Cause of death \\
\hline Case 1 & Female & 4 & 6 & Pulmonary hemorrhage \\
Case 2 & Male & 140 & 143 & Alveolar capillary dysplasia, respiratory failure \\
Case 3 & Male & 49 & 90 & Jejunal perforation, septic shock \\
Case 4 & Female & 7 & 49 & Tension pneumothorax \\
Case 5 & Male & 40 & 68 & Persistent pulmonary hypertension, multi-organ failure \\
Case 6 & Male & 11 & 20 & Persistent pulmonary hypertension, bowel ischemia, multi-organ failure \\
Case 7 & Female & 26 & 32 & Sepsis \\
\hline
\end{tabular}

Table 2. Baseline characteristics of patients treated with extracorporeal membrane oxygenation

\begin{tabular}{|c|c|c|c|c|}
\hline Characteristic & Overall $(n=51)$ & Survivors $(n=16)$ & Nonsurvivors $(\mathrm{n}=35)$ & p-value \\
\hline Male sex & $33(64.7)$ & $12(75.0)$ & $21(60.0)$ & 0.469 \\
\hline Gestational age (day) & $270.3 \pm 8.8$ & $267.1 \pm 7.2$ & $271.7 \pm 9.2$ & 0.083 \\
\hline Birth weight $(\mathrm{kg})$ & $3.1 \pm 0.5$ & $3.1 \pm 0.5$ & $3.1 \pm 0.4$ & 0.659 \\
\hline Prematurity & $1(2.0)$ & $0(0.0)$ & $1(2.9)$ & 0.999 \\
\hline Low birth weight & $7(13.7)$ & $2(12.5)$ & $5(14.3)$ & 0.999 \\
\hline Congenital anomaly & $9(17.6)$ & $1(6.2)$ & $8(22.9)$ & 0.295 \\
\hline Congenital heart disease & $9(17.6)$ & $3(18.8)$ & $6(17.1)$ & 0.990 \\
\hline Cesarean section & $37(72.5)$ & $11(68.8)$ & $26(74.3)$ & 0.942 \\
\hline Left CDH & $39(76.5)$ & $10(62.5)$ & 29 (82.9) & 0.217 \\
\hline Liver herniation & $32(69.6)$ & $8(61.5)$ & $24(72.7)$ & 0.699 \\
\hline O/E LHR, longitudinal (\%) & $41.5 \pm 17.3$ & $38.8 \pm 10.4$ & $43.0 \pm 20.1$ & 0.543 \\
\hline O/E LHR tracing $(\%)$ & $42.7 \pm 17.5$ & $42.0 \pm 11.9$ & $43.1 \pm 20.2$ & 0.875 \\
\hline Apgar score at $1 \mathrm{~min}$ & $4.9 \pm 1.51$ & $5.1 \pm 1.57$ & $4.8 \pm 1.5$ & 0.528 \\
\hline Apgar score at $5 \mathrm{~min}$ & $6.9 \pm 1.18$ & $7.2 \pm 1.22$ & $6.7 \pm 1.2$ & 0.214 \\
\hline
\end{tabular}

Values are presented as number (\%) or mean \pm standard deviation.

$\mathrm{CDH}$, congenital diaphragmatic hernia; O/E LHR, observed/expected lung-to-head ratio.

Table 3. Clinical variables before ECMO initiation

\begin{tabular}{|c|c|c|c|c|}
\hline Variable & Overall $(n=51)$ & Survivors $(n=16)$ & Nonsurvivors $(\mathrm{n}=35)$ & $p$-value \\
\hline Initial lactate level $(\mathrm{mmol} / \mathrm{dL})$ & $3.8(2.4-7.7)$ & $3.2(2.2-3.9)$ & $5.8(2.4-8.9)$ & 0.048 \\
\hline Peak lactate level (mmol/dL) & $5.8(3.9-10.8)$ & $5.2(4.1-6.3)$ & $7.9(3.9-13.9)$ & 0.023 \\
\hline \multicolumn{5}{|l|}{ Oxygenation index } \\
\hline $6 \mathrm{hr}$ after birth & $48.5(26.3-61.4)$ & $24.1(15.6-33.4)$ & $55.2(36.7-76.6)$ & 0.002 \\
\hline 12 hr after birth & $36.4(24.6-51.4)(n=32)$ & $29.4(23.4-36.6)(n=14)$ & $49.3(32.6-70.0)(n=18)$ & 0.024 \\
\hline $1 \mathrm{hr}$ before ECMO & $78.3(51.0-111.0)$ & $51.0(38.5-67.0)$ & $90.0(62.5-133.5)$ & 0.005 \\
\hline Pulmonary hypertension & $50(98.0)$ & $15(93.8)$ & $35(100.0)$ & 0.685 \\
\hline RL shunt through a PDA & $35(68.6)$ & $12(75.0)$ & $23(65.7)$ & 0.507 \\
\hline Inhaled nitric oxide & $49(96.1)$ & $15(93.8)$ & $34(97.1)$ & 0.999 \\
\hline CPCR & $7(13.7)$ & $1(6.2)$ & $6(17.1)$ & 0.542 \\
\hline
\end{tabular}

Values are presented as median (interquartile range) or number (\%), unless otherwise stated.

ECMO, extracorporeal membrane oxygenation; RL, right to left; PDA, patent ductus arteriosus; CPCR, cardiopulmonary cerebral resuscitation.

hours after birth (24.1 versus $55.2, \mathrm{p}=0.002)$ and at 1 hour before ECMO initiation (51.0 versus 90.0, $\mathrm{p}=0.005$ ) were significantly lower in the survivor group than in the nonsurvivor group.

\section{Extracorporeal membrane oxygenation-related variables}

Table 4 describes the variables related to the ECMO runs. The time to ECMO initiation was not significantly different between the groups $(\mathrm{p}=0.239)$. The mean dura- 
Table 4. Variables related to ECMO runs

\begin{tabular}{|c|c|c|c|c|}
\hline Variable & Overall $(n=51)$ & Survivors $(n=16)$ & Nonsurvivors $(\mathrm{n}=35)$ & p-value \\
\hline ECMO initiation (hour after birth) & $17.0(7.5-30.0)$ & $28.5(15.8-35.0)$ & $12.0(7.0-23.5)$ & 0.239 \\
\hline Total ECMO run (day) & $8.0(4.9-21.4)$ & $6.1(4.9-7.2)$ & $15.1(5.0-29.6)$ & 0.039 \\
\hline Veno-arterial ECMO & $27(52.9)$ & $11(68.8)$ & $16(45.7)$ & 0.220 \\
\hline Veno-venous ECMO & $24(47.1)$ & $5(31.2)$ & $19(37.3)$ & 0.190 \\
\hline Repair timing & & & & $<0.001$ \\
\hline Before ECMO & $3(6.5)$ & $2(12.5)$ & $1(3.3)$ & \\
\hline During ECMO & $36(78.3)$ & $7(43.8)$ & $29(96.7)$ & \\
\hline After ECMO & $7(15.2)$ & $7(43.8)$ & 0 & \\
\hline Patch repair & $36(78.3)$ & $13(81.2)$ & $23(76.7)$ & 0.999 \\
\hline Major bleeding & $22(43.1)$ & $2(12.5)$ & $20(57.1)$ & 0.007 \\
\hline Major surgical bleeding & $15(29.4)$ & $1(6.2)$ & $14(40.0)$ & 0.034 \\
\hline Major medical bleeding & $13(25.5)$ & $1(6.2)$ & $12(34.3)$ & 0.074 \\
\hline
\end{tabular}

Values are presented as median (interquartile range) or number (\%), unless otherwise stated.

$\mathrm{ECMO}$, extracorporeal membrane oxygenation.

Table 5. Univariate and multivariate analyses of the risk of in-hospital mortality

\begin{tabular}{|c|c|c|c|c|}
\hline \multirow{2}{*}{ Variable } & \multicolumn{2}{|c|}{ Univariate } & \multicolumn{2}{|c|}{ Multivariate } \\
\hline & OR $(95 \% \mathrm{Cl})$ & p-value & OR $(95 \% \mathrm{CI})$ & p-value \\
\hline Male sex & $0.50(0.12-1.79)$ & 0.30 & & \\
\hline Low birth weight & $1.16(0.22-9.09)$ & 0.86 & & \\
\hline Congenital anomaly & $4.35(0.71-86.50)$ & 0.18 & & \\
\hline Left CDH & $0.34(0.09-1.33)$ & 0.12 & & \\
\hline Liver herniation & $1.67(0.41-6.67)$ & 0.46 & & \\
\hline Peak lactate level & $1.20(1.04-1.47)$ & 0.03 & $1.02(0.82-1.29)$ & 0.845 \\
\hline Oxygenation index $1 \mathrm{hr}$ before ECMO & $1.03(1.01-1.05)$ & 0.01 & $1.02(1.01-1.04)$ & 0.055 \\
\hline Multidisciplinary treatment & $0.16(0.04-0.58)$ & 0.006 & $0.29(0.06-1.35)$ & 0.120 \\
\hline Patch repair & $0.76(0.14-3.33)$ & 0.72 & & \\
\hline Veno-arterial ECMO & $0.38(0.1-1.30)$ & 0.13 & & \\
\hline Major bleeding & $9.33(2.18-65.43)$ & 0.007 & $5.84(1.06-47.8)$ & 0.059 \\
\hline Major medical bleeding & $8.33(1.33-166.67)$ & 0.06 & & \\
\hline Major surgical bleeding & $10.00(1.72-191.10)$ & 0.03 & & \\
\hline
\end{tabular}

$\mathrm{OR}$, odds ratio; $\mathrm{Cl}$, confidence interval; $\mathrm{CDH}$, congenital diaphragmatic hernia; $\mathrm{ECMO}$, extracorporeal membrane oxygenation.

tion of ECMO support was shorter in the survivor group than in the nonsurvivor group (6.5 versus 15.1 days, $\mathrm{p}=0.039)$. Overall, 27 patients $(52.9 \%)$ were treated using veno-arterial ECMO, and there was no significant difference in the distribution of the ECMO mode between groups. Seven patients (43.8\%) in the survivor group were weaned from ECMO without hernia repair. Major surgical bleeding more frequently occurred in the nonsurvivor group $(\mathrm{p}=0.034)$.

\section{Factors associated with hospital survival}

Table 5 shows the risk factors associated with in-hospital mortality. In the univariate analysis using logistic regression, peak lactate level, OI at 1 hour before ECMO initia- tion, and major surgical bleeding were factors associated with the risk of in-hospital mortality, whereas the multidisciplinary team approach had a protective effect against in-hospital mortality. In the multivariate logistic regression analysis, the OI at 1 hour before ECMO initiation (odds ratio, 1.02; 95\% confidence interval, 1.01-1.05, p=0.05) and major bleeding (odds ratio, 5.84; 95\% confidence interval, 1.06-47.8, $\mathrm{p}=0.05$ ) were identified as independent risk factors for in-hospital mortality.

\section{Follow-up}

Sixteen survivors were followed up for a mean duration of 53.6 months. There were no late deaths. No patient required at-home oxygen therapy. Hypoplasia of the affected 
lung and chest asymmetry were observed on follow-up chest roentgenography in all patients. Two patients showed stroke in the right hemisphere of the brain. Both patients had veno-arterial ECMO support.

\section{Discussion}

$\mathrm{CDH}$ is the most common indication for ECMO in cases of neonatal respiratory failure in the ELSO database. However, patients with $\mathrm{CDH}$ requiring ECMO continue to have the lowest rate of survival to discharge (50\%) compared with those with other indications, such as meconium aspiration syndrome (93\%) or persistent pulmonary hypertension of newborns $(74 \%)[3,4]$. This is attributable to the change in the risk profile of patients with $\mathrm{CDH}$ requiring ECMO, which is a high-risk group that has shown a greater tendency than other groups to undergo ECMO in recent years [5]. However, even considering the severely detrimental conditions in patients with $\mathrm{CDH}$ requiring ECMO, a $50 \%$ survival rate is suboptimal. Efforts have been made to improve the outcomes by standardizing the management, involving a multidisciplinary team, and individualizing the treatment [3]. In this study that used single-center data from the start of the ECMO program for $\mathrm{CDH}$ in 2008, the overall rate of successful weaning from ECMO was $45.1 \%$ and the hospital survival rate was $31.4 \%$.

In our study, antenatal fetal ultrasound data showed no significant difference between the survivor and nonsurvivor groups. Several studies have been published on the evaluation of the disease severity of $\mathrm{CDH}$ before birth. The lung-to-head ratio on fetal ultrasonography and the lung volume measured on magnetic resonance imaging are known to have predictive value for survival and ECMO requirement in patients with $\mathrm{CDH}[6,7]$. However, a study using the ELSO database failed to identify predictive factors in patients already treated with ECMO [8]. It seems that antenatal measurements can predict the disease severity and the need for ECMO, but might not be associated with mortality after ECMO initiation.

Clinical variables before ECMO initiation, such as the OI and arterial blood gas levels, showed significant differences between the survivor and nonsurvivor groups. The multivariate analysis revealed that the OI at 1 hour before ECMO initiation was associated with the risk of mortality. A recent study by Guner et al, [9], who attempted to develop a risk model for mortality in patients with $\mathrm{CDH}$ treated with ECMO, also showed similar results. In their study, pre-ECMO blood gas level and pre-ECMO ventilator settings and comorbidities affected the survival of patients treated with ECMO. Although worsening of clinical variables predicts a higher risk of mortality in patients treated with ECMO, it should not preclude the utilization of extracorporeal support, as ECMO is usually the only rescue treatment available for rapid cardiopulmonary collapse.

The early application of ECMO should be considered before irreversible tissue damage occurs, which can be reflected by a high OI and high lactate levels. However, the optimal timing of ECMO initiation is controversial. As ECMO is a highly invasive treatment and carries the risk of serious neurologic comorbidities and bleeding complications, physicians tend to be reluctant to initiate ECMO. Several guidelines suggest clinical criteria for the indications of ECMO; however, no consensus has been reached $[4,10]$. The ELSO guidelines emphasize the need for early initiation of ECMO for preoperative stabilization. However, there are currently no data on the impact of the timing of ECMO initiation. Late ECMO initiation may increase the risk of significant barotrauma to the lung and right heart failure [11]. A study revealed increased survival in patients with early referral (within 24 hours) to the ECMO center [12]. Therefore, timely administration of extracorporeal support is crucial in the treatment of $\mathrm{CDH}$.

Veno-arterial ECMO via the cervical approach has been the traditional method of cannulation in neonates worldwide. It has the advantages of providing hemodynamic support, using a smaller cannula, and being less influenced by the position of cannulae than veno-venous ECMO. With the development of dual-lumen catheters, the veno-venous mode of ECMO has been introduced in the treatment of $\mathrm{CDH}$ in neonates [13]. This mode can reduce the neurologic embolism risk by not interrupting the carotid artery flow. Veno-venous ECMO can also provide oxygenated blood to the pulmonary vasculature and myocardium and is considered more physiologic than the veno-arterial mode. In a study using the ELSO Registry database, the mortality and complications rates were comparable between the veno-arterial and veno-venous groups. Renal complications and inotropic use were prevalent in the veno-venous group. However, neurologic morbidity was higher in the veno-arterial group [14]. At our center, although the veno-venous mode was introduced and widely applied for a certain period, the veno-arterial mode has re-entered use, mainly because of the lack of supply of dual-lumen cannulae and the requirement for hemodynamic support in patients with ventricular dysfunction combined with pulmonary hypertension. There was no significant difference in the ECMO mode between survivors and nonsurvivors. 
This study showed that delayed repair of the diaphragm after weaning from ECMO showed a survival benefit with low bleeding complications. Similarly, the multivariate analysis showed that major bleeding was a significant risk factor for in-hospital mortality. However, the appropriate timing of hernia repair related to ECMO application remains controversial. The strategies are mainly divided into early repair while on ECMO and delayed repair after ECMO decannulation. Early repair provides favorable conditions for the surgeon, with less tissue edema and the possibility of reversing the pathophysiology of persistent pulmonary hypertension [15]. However, patients with ECMO support require systemic anticoagulation, which increases the risk of major surgical bleeding. In contrast, late repair after decannulation has the advantage of fewer bleeding complications, which can promote the reversibility of pulmonary hypertension [16]. One consideration regarding the late repair strategy is that patients who fail to be weaned from ECMO lose the opportunity to undergo diaphragmatic hernia repair. Anatomic repair of the diaphragm may help attenuate pulmonary hypertension, as mentioned earlier. An attempt to repair the hernia during ECMO should be considered as the duration of ECMO becomes longer. Further studies are warranted to establish the appropriate timing of attempting repair in cases of unsuccessful weaning from ECMO.

A total of 16 patients who survived to discharge were followed up until recently. No mortality occurred; however, ischemic stroke was found in 2 patients. Patients with $\mathrm{CDH}$ treated with ECMO have been reported to have poor neurologic outcomes. In a study of 12 ECMO survivors, 8 patients (67\%) showed neurologic delays and a recent study from the Mayo Clinic also reported a high prevalence of chronic complications in ECMO survivors $[17,18]$. In a study performed in the United Kingdom, which reviewed 73 neonates who were treated with ECMO for $\mathrm{CDH}, 42$ patients were discharged home, but only 27 patients survived to the age of 1 year. Comorbidities were also common in long-term survivors, of whom 13 (48\%) had respiratory symptoms, 16 (59\%) had gastrointestinal problems, and 6 (19\%) had severe neurodevelopmental problems [19]. Persistent pulmonary hypertension has been observed in patients who were successfully weaned from ECMO but died before discharge.

In conclusion, the survival rate of patients with $\mathrm{CDH}$ treated with ECMO is suboptimal. Timely application of ECMO is crucial for better survival outcomes.

\section{Conflict of interest}

No potential conflict of interest relevant to this article was reported.

\section{ORCID}

Wooseok Choi: https://orcid.org/0000-0002-7073-0442

Won Chul Cho: https://orcid.org/0000-0002-3858-6516

Eun Seok Choi: https://orcid.org/0000-0003-2480-126X

Tae-Jin Yun: https://orcid.org/0000-0002-0336-1720

Chun Soo Park: https://orcid.org/0000-0001-8718-8904

\section{References}

1. Logan JW, Cotten CM, Goldberg RN, Clark RH. Mechanical ventilation strategies in the management of congenital diaphragmatic hernia. Semin Pediatr Surg 2007;16:115-25.

2. Kays DW. ECMO in CDH: is there a role? Semin Pediatr Surg 2017; 26:166-70

3. Guner Y, Jancelewicz T, Di Nardo M, et al. Management of congenital diaphragmatic hernia treated with extracorporeal life support: interim guidelines consensus statement from the Extracorporeal Life Support Organization. ASAIO J 2021;67:113-20.

4. Barbaro RP, Paden ML, Guner YS, et al. Pediatric Extracorporeal Life Support Organization Registry International Report 2016. ASAIO J 2017;63:456-63.

5. Guner YS, Delaplain PT, Zhang L, et al. Trends in mortality and risk characteristics of congenital diaphragmatic hernia treated with extracorporeal membrane oxygenation. ASAIO J 2019;65:509-15.

6. Mahieu-Caputo D, Sonigo P, Dommergues M, et al. Fetal lung volume measurement by magnetic resonance imaging in congenital diaphragmatic hernia. BJOG 2001;108:863-8.

7. Aspelund G, Fisher JC, Simpson LL, Stolar CJ. Prenatal lung-head ratio: threshold to predict outcome for congenital diaphragmatic hernia. J Matern Fetal Neonatal Med 2012;25:1011-6.

8. Heiss KF, Clark RH. Prediction of mortality in neonates with congenital diaphragmatic hernia treated with extracorporeal membrane oxygenation. Crit Care Med 1995;23:1915-9.

9. Guner YS, Nguyen DV, Zhang L, et al. Development and validation of extracorporeal membrane oxygenation mortality-risk models for congenital diaphragmatic hernia. ASAIO J 2018;64:785-94.

10. Snoek KG, Reiss IK, Greenough A, et al. Standardized postnatal management of infants with congenital diaphragmatic hernia in Europe: the CDH EURO Consortium Consensus: 2015 update. Neonatology 2016;110:66-74.

11. Rafat N, Schaible T. Extracorporeal membrane oxygenation in congenital diaphragmatic hernia. Front Pediatr 2019;7:336.

12. Schaible T, Hermle D, Loersch F, Demirakca S, Reinshagen K, Varn- 
holt V. A 20-year experience on neonatal extracorporeal membrane oxygenation in a referral center. Intensive Care Med 2010;36:122934.

13. Heiss KF, Clark RH, Cornish JD, et al. Preferential use of venovenous extracorporeal membrane oxygenation for congenital diaphragmatic hernia. J Pediatr Surg 1995;30:416-9.

14. Guner YS, Khemani RG, Qureshi FG, et al. Outcome analysis of neonates with congenital diaphragmatic hernia treated with venovenous vs venoarterial extracorporeal membrane oxygenation. J Pediatr Surg 2009;44:1691-701.

15. Fallon SC, Cass DL, Olutoye OO, et al. Repair of congenital diaphragmatic hernias on extracorporeal membrane oxygenation (ECMO): does early repair improve patient survival? J Pediatr Surg 2013;48:1172-6
16. Partridge EA, Peranteau WH, Rintoul NE, et al. Timing of repair of congenital diaphragmatic hernia in patients supported by extracorporeal membrane oxygenation (ECMO). J Pediatr Surg 2015;50:260-2.

17. McGahren ED, Mallik K, Rodgers BM. Neurological outcome is diminished in survivors of congenital diaphragmatic hernia requiring extracorporeal membrane oxygenation. J Pediatr Surg 1997;32:121620.

18. Bojanic K, Woodbury JM, Cavalcante AN, et al. Congenital diaphragmatic hernia: outcomes of neonates treated at Mayo Clinic with and without extracorporeal membrane oxygenation. Paediatr Anaesth 2017;27:314-21.

19. Davis PJ, Firmin RK, Manktelow B, et al. Long-term outcome following extracorporeal membrane oxygenation for congenital diaphragmatic hernia: the UK experience. J Pediatr 2004;144:309-15. 